\title{
EDITORIAL
}

\section{Jumbled NETs Promote Vasculitis}

\author{
Marko Radic ${ }^{1}$ and Mariana J. Kaplan ${ }^{2}$
}

Drug-induced autoimmunity provides a fascinating glimpse of the environment's contribution to the development of anti-self reactivity. After a period of treatment, certain drugs induce, in a subset of individuals, clinical signs that are typically found only in autoimmune disorders. The clinical features of drug-induced autoimmunity are a reminder that genetic predisposition to self reactivity usually needs a trigger from the environment in order to initiate a self-sustaining vicious circle. Notably, the cessation of drug therapy typically brings about the resolution of the autoimmune manifestations. Thus, studies of drug-induced autoimmunity provide an opportunity to learn not only about environmental triggers that lead to a loss of tolerance, but also about how a disruption of a specific stimulus may allow the immune system to return to a previous state of nonautoimmune homeostasis.

Historically, hydralazine, a hypertension medication, was the first to be reported to induce systemic lupus erythematosus (SLE) in up to $24-50 \%$ of the patients taking it (1). This medication can also induce symptoms of vasculitis. Other medications, such as procainamide, chlorpromazine, penicillamine, and sulfasalazine, were observed to induce SLE and vasculitis. The discovery of drugs that induce autoimmunity prompted researchers to examine possible mechanisms. Various hypotheses have been proposed, including that the drug structure mimics the autoantigen, that the drug chemically modifies the autoantigen structure, that it inhibits DNA methylation thereby leading to perturbed $\mathrm{T}$ cell regulation, that it alters mechanisms of antigen presentation or

\footnotetext{
Dr. Radic's research related to this topic was supported by the Lupus Research Institute of New York.

${ }^{1}$ Marko Radic, PhD: University of Tennessee Health Science Center, Memphis; ${ }^{2}$ Mariana J. Kaplan, MD: University of Michigan Medical School, Ann Arbor.

Address correspondence to Marko Radic, PhD, Department of Microbiology, Immunology and Biochemistry, University of Tennessee Health Science Center, 858 Madison Avenue, Suite 211, Memphis, TN 38163. E-mail: mradic@uthsc.edu.

Submitted for publication June 18, 2012; accepted in revised form June 28, 2012.
}

lymphocyte activation, or that the drug is converted into chemically more reactive compounds in the body. However, thus far the testing of the proposed mechanisms has been difficult, and few conclusions have been put forth to explain how drugs induce autoreactivity (2).

The article by Nakazawa et al in this issue of Arthritis \& Rheumatism (3) presents a new twist on drug-mediated induction of autoimmunity. The authors examined the connection between propylthiouracil (PTU), a drug that is commonly prescribed for hyperthyroidism or Graves' disease, and the subsequent development of a form of vasculitis that is associated with the production of autoantibodies to myeloperoxidase (MPO), an enzyme stored in neutrophil granules. The authors explored the role of neutrophil cell death in PTU-induced autoimmunity. It is of interest to view the findings of Nakazawa et al in the context of previous observations on the role of PTU in the induction of vasculitis and other autoimmune manifestations.

PTU is a thionamide drug that, since 1947, has been prescribed to millions of patients with elevated levels of thyroid hormone. Graves' disease is frequently caused by autoantibodies that bind the thyroidstimulating hormone receptor and lead to the overproduction of thyroid hormone. PTU blocks a key enzyme in the synthesis of T4 from its precursor thyroglobulin. The thyroid gland secretes $\mathrm{T} 4$ following its iodination on tyrosine by thyroid peroxidase (TPO). PTU inhibits oxidation of iodide by TPO and therefore effectively limits the production of thyroid hormone. TPO is structurally related to lactoperoxidase and MPO. The similarity between members of this family of proteins is so high that MPO can be used in enzyme studies as a stand-in for TPO (4), and structural similarity between MPO and TPO was exploited to express chimeric molecules and map antibody epitopes on TPO (5). In that light, it is perhaps not surprising that PTU also inhibits the enzymatic reactions of MPO (6).

The side effects of PTU in patients with hyperthyroidism include the production of antibodies to MPO, 
and some individuals with PTU-induced anti-MPO antibodies progress to exhibit additional symptoms, including cutaneous vasculitis, crescentic glomerulonephritis, and agranulocytosis (7-9). In idiopathic vasculitis, the tight correlation between anti-MPO autoantibodies and disease was previously observed. Small-vessel vasculitis is commonly related to the presence of antineutrophil cytoplasmic antibodies (ANCAs). More specifically, anti-MPO antibodies serve as markers for the diagnosis of microscopic polyangiitis, while anti-proteinase 3 antibodies are considered markers for the diagnosis of granulomatosis with polyangiitis (Wegener's) (10).

Antibodies to MPO are considered to be directly involved in the pathogenesis of vascular inflammation, since mice immunized with MPO develop systemic vasculitis with crescentic glomerulonephritis and pulmonary hemorrhage (11). The symptoms can be transferred from immunized mice to naive animals by transfer of purified anti-MPO IgG. The necessary contribution of neutrophils in this model of ANCA-associated vasculitis (AAV) was shown by the reduced severity of symptoms in mice whose neutrophils were depleted prior to infusion of the pathogenic autoantibodies (12). Incubation of neutrophils with ANCA induces degranulation and reactive oxygen production, thus suggesting how antiMPO ANCA-induced inflammation could give rise to vasculitis (13). In WKY rats, immunizations with human MPO have been optimized to result in a $100 \%$ incidence of crescentic glomerulonephritis and lung hemorrhage (14). It has thus been confirmed that anti-MPO antibodies can, on a susceptible genetic background, lead to the most severe manifestations of systemic vasculitis.

In the context of the prior studies, Nakazawa et al proposed the attractive hypothesis that PTU perturbs neutrophil cell death in such a manner as to induce anti-MPO antibodies and produce symptoms of vasculitis. The results of previous studies had suggested that apoptotic cell death provides access to nuclear autoantigens, and that an immune response to apoptotic cells may lead to systemic autoimmune disease $(15,16)$. However, the question of whether other forms of cell death provide access to autoantigens has been less thoroughly examined.

A distinct and potentially relevant form of cell death leads to the release of nuclear chromatin from granulocytes (17). The released chromatin is referred to as a neutrophil extracellular trap (NET) because it may serve to immobilize infectious microbes (18). NETs are thought to be an innate response to infections because they organize neutrophil granule components and contribute to the neutrophil's bactericidal functions.
The production of NETs occurs as a result of a unique form of cell death called NETosis (17). NETosis appears to depend on the cell's ability to produce reactive oxygen species, including superoxide, and various oxidation products generated by MPO (19). Interestingly, ANCAs induce NETs in vitro, and NETs were detected in kidney biopsy specimens from patients with small-vessel vasculitis (20). Thus, it was reasonable to predict that NETosis could play a role in PTU-induced vasculitis.

Nakazawa et al (3) provide exciting data showing that PTU inhibits full release of NET chromatin and results in a more compact NET scaffold. This is despite the fact that PTU-treated NETs contained normal levels of deiminated histone $\mathrm{H} 3$, a histone modification that precedes and is required for NET release (21). The altered morphology of PTU NETs correlated with an increased resistance to nucleolytic degradation; thus, PTU presumably lengthens the NET's half-life in vivo. Furthermore, the authors reported that immunization of WKY rats with NETs formed in the presence of PTU resulted in the production of anti-MPO ANCA and pulmonary hemorrhage. To test whether oral administration of PTU affects the induction of vasculitis in vivo, Nakazawa et al added PTU to the rats' drinking water and induced NETosis by weekly intraperitoneal injections of phorbol myristate acetate, a potent NET agonist. The rats receiving both treatments had more anti-MPO ANCA, greater pulmonary hemorrhage, worse renal function, and a higher incidence of glomerulonephritis than the rats receiving either chemical alone (3).

The immediate contribution of Nakazawa et al includes the testing of a possible mechanism of PTUinduced MPO AAV and the development of a new animal model of this form of vasculitis. Nonetheless, important questions remain to be addressed. Do the anti-MPO antibodies generated by PTU/NET immunizations have the capacity to transfer disease to a naive animal? Is MPO indeed the target of PTU, and is the altered structure of PTU-treated NETs due to the inhibition of MPO activity? Would immunization with PTU-MPO complexes induce anti-MPO autoantibodies, or is the presence of DNAse-resistant NETs important in stimulating the anti-MPO response? The work by Nakazawa et al also raises hopes that we will learn more about environmental contributions to autoimmunity. Do infections or an autoimmune genetic background that leads to exaggerated NETosis contribute to the initial manifestations of MPO AAV? Do other drugs that induce autoimmune manifestations also affect NETosis? It may be important to examine drugs 
that are known to induce agranulocytosis, since one way to deplete neutrophils is by inducing excessive NETosis. Potential candidates are chlorpromazine, procainamide, hydralazine, and sulfasalazine.

The insights provided by Nakazawa et al also have broader implications. Other studies have suggested that NET-associated autoantigens are important stimuli in autoimmunity, as MPO ANCAs are only one of many autoantibodies directed at NET components. An interesting comparison is provided by antibodies to deiminated histones, a potentially useful marker for neutrophil responses to inflammatory stimuli (22). IgG from patients with Felty's syndrome exhibit a consistent preference for deiminated histones over nondeiminated histones, suggesting that NETs are involved in breaking immune tolerance in this disorder (23). NET components could directly stimulate the immune system, as complexes between chromatin and LL37, a neutrophil granule component that tightly associates with NETs, stimulate activation of plasmacytoid dendritic cells and secretion of type I interferon (24). The presence of autoantibodies to NET components, just like the administration of PTU, may protect NETs against degradation, suggesting that, in autoimmunity, these chromatin matrices persist for a longer time (25). This may be amplified by the tendency of an aberrant subset of neutrophils for enhanced spontaneous NETosis in disorders such as SLE and psoriasis (26,27), conditions that can be triggered by medications. Aberrant NET formation can potentially promote autoreactivity in other ways, including the enhanced activation of $\mathrm{T}$ cells (28). These studies support the concept that NETosis is a relevant source of autoantigens in autoimmunity. This new paradigm holds promise for a better understanding of the pathogenic mechanisms leading to the loss of tolerance and for the discovery of new therapies for various autoimmune diseases.

\section{AUTHOR CONTRIBUTIONS}

Drs. Radic and Kaplan drafted the article, revised it critically for important intellectual content, and approved the final version to be published.

\section{REFERENCES}

1. Perry HM Jr, Schroeder HA. Syndrome simulating collagen disease caused by hydralazine (Apresoline). J Am Med Assoc 1954;154:670-3.

2. Deng $\mathrm{C}$, Lu Q, Zhang Z, Rao T, Attwood J, Yung R, et al. Hydralazine may induce autoimmunity by inhibiting extracellular signal-regulated kinase pathway signaling. Arthritis Rheum 2003; 48:746-56.

3. Nakazawa D, Tomaru U, Suzuki A, Masuda S, Hasegawa R,
Kobayashi T, et al. Abnormal conformation and impaired degradation of propylthiouracil-induced neutrophil extracellular traps: implications of disordered neutrophil extracellular traps in a rat model of myeloperoxidase antineutrophil cytoplasmic antibodyassociated vasculitis. Arthritis Rheum 2012;64:3779-87.

4. Taurog A, Dorris ML. Myeloperoxidase-catalyzed iodination and coupling. Arch Biochem Biophys 1992;296:239-46.

5. Nishikawa T, Nagayama Y, Seto P, Rapoport B. Human thyroid peroxidase-myeloperoxidase chimeric molecules: tools for the study of antigen recognition by thyroid peroxidase autoantibodies. Endocrinology 1993;133:2496-501.

6. Lee E, Miki Y, Katsura H, Kariya K. Mechanism of inactivation of myeloperoxidase by propylthiouracil. Biochem Pharmacol 1990; 39:1467-71.

7. Amrhein JA, Kenny FM, Ross D. Granulocytopenia, lupus-like syndrome, and other complications of propylthiouracil therapy. J Pediatr 1970;76:54-63.

8. Dolman KM, Gans RO, Vervaat TJ, Zevenbergen G, Maingay D, Nikkels RE, et al. Vasculitis and antineutrophil cytoplasmic autoantibodies associated with propylthiouracil therapy. Lancet 1993; 342:651-2.

9. Sera N, Ashizawa K, Ando T, Abe Y, Ide A, Usa T, et al. Treatment with propylthiouracil is associated with appearance of antineutrophil cytoplasmic antibodies in some patients with Graves' disease. Thyroid 2000;10:595-9.

10. Csernok E, Lamprecht P, Gross WL. Clinical and immunological features of drug-induced and infection-induced proteinase 3-antineutrophil cytoplasmic antibodies and myeloperoxidaseantineutrophil cytoplasmic antibodies and vasculitis. Curr Opin Rheumatol 2010;22:43-8.

11. Xiao H, Heeringa P, Hu P, Liu Z, Zhao M, Aratani Y, et al. Antineutrophil cytoplasmic autoantibodies specific for myeloperoxidase cause glomerulonephritis and vasculitis in mice. J Clin Invest 2002;110:955-63.

12. Xiao H, Heeringa P, Liu Z, Huugen D, Hu P, Maeda N, et al. The role of neutrophils in the induction of glomerulonephritis by anti-myeloperoxidase antibodies. Am J Pathol 2005;167:39-45.

13. Falk RJ, Terrell RS, Charles LA, Jennette JC. Anti-neutrophil cytoplasmic autoantibodies induce neutrophils to degranulate and produce oxygen radicals in vitro. Proc Natl Acad Sci U S A 1990;87:4115-9.

14. Little MA, Smyth L, Salama AD, Mukherjee S, Smith J, Haskard D, et al. Experimental autoimmune vasculitis: an animal model of anti-neutrophil cytoplasmic autoantibody-associated systemic vasculitis. Am J Pathol 2009;174:1212-20.

15. Casciola-Rosen LA, Anhalt G, Rosen A. Autoantigens targeted in systemic lupus erythematosus are clustered in two populations of surface structures on apoptotic keratinocytes. J Exp Med 1994;179:1317-30.

16. Radic M, Marion T, Monestier M. Nucleosomes are exposed at the cell surface in apoptosis. J Immunol 2004;172:6692-700.

17. Fuchs TA, Abed U, Goosmann C, Hurwitz R, Schulze I, Wahn V, et al. Novel cell death program leads to neutrophil extracellular traps. J Cell Biol 2007;176:231-41.

18. Brinkmann V, Reichard U, Goosmann C, Fauler B, Uhlemann Y, Weiss DS, et al. Neutrophil extracellular traps kill bacteria Science 2004;303:1532-5.

19. Kirchner T, Moller S, Klinger M, Solbach W, Laskay T, Behnen M. The impact of various reactive oxygen species on the formation of neutrophil extracellular traps. Mediators Inflamm 2012;2012: 849136.

20. Kessenbrock K, Krumbholz M, Schonermarck U, Back W, Gross WL, Werb Z, et al. Netting neutrophils in autoimmune smallvessel vasculitis. Nat Med 2009;15:623-5.

21. Li P, Li M, Lindberg MR, Kennett MJ, Xiong N, Wang Y. PAD4 is essential for antibacterial innate immunity mediated by neutrophil extracellular traps. J Exp Med 2010;207:1853-62. 
22. Neeli I, Khan SN, Radic M. Histone deimination as a response to inflammatory stimuli in neutrophils. J Immunol 2008;180:1895-902.

23. Dwivedi N, Upadhyay J, Neeli I, Khan S, Pattanaik D, Myers L, et al. Felty's syndrome autoantibodies bind to deiminated histones and neutrophil extracellular chromatin traps. Arthritis Rheum 2012;64:982-92.

24. Lande R, Ganguly D, Facchinetti V, Frasca L, Conrad C, Gregorio $\mathrm{J}$, et al. Neutrophils activate plasmacytoid dendritic cells by releasing self-DNA-peptide complexes in systemic lupus erythematosus. Sci Transl Med 2011;3:73ra19.

25. Hakkim A, Furnrohr BG, Amann K, Laube B, Abed UA, Brinkmann V, et al. Impairment of neutrophil extracellular trap degradation is associated with lupus nephritis. Proc Natl Acad Sci U S A 2010;107:9813-8.

26. Villanueva E, Yalavarthi S, Berthier CC, Hodgin JB, Khandpur R, Lin AM, et al. Netting neutrophils induce endothelial damage, infiltrate tissues, and expose immunostimulatory molecules in systemic lupus erythematosus. J Immunol 2011;187:538-52.

27. Lin AM, Rubin CJ, Khandpur R, Wang JY, Riblett M, Yalavarthi S, et al. Mast cells and neutrophils release IL-17 through extracellular trap formation in psoriasis. J Immunol 2011;187:490-500.

28. Tillack K, Breiden P, Martin R, Sospedra M. T lymphocyte priming by neutrophil extracellular traps links innate and adaptive immune responses. J Immunol 2012;188:3150-9. 